\title{
Dramatic radiographic response resulting in cerebrospinal fluid rhinorrhea associated with sunitinib therapy in recurrent atypical meningioma: case report
}

\author{
Amol Raheja, MBBS, ${ }^{1}$ Howard Colman, MD, PhD, ${ }^{1}$ Cheryl A. Palmer, MD, ${ }^{2}$ and \\ William T. Couldwell, MD, PhD'1 \\ Departments of ${ }^{1}$ Neurosurgery and ${ }^{2}$ Pathology, University of Utah, Salt Lake City, Utah
}

Sunitinib is a multiple tyrosine kinase inhibitor with antiangiogenic, cytostatic, and antimigratory activity for meningiomas. A recent clinical trial of sunitinib for treatment of recurrent Grade II and III meningiomas suggested potential efficacy in this population, but only 2 patients exhibited significant radiographic response with tumor volume reduction. The authors illustrate another such case and discuss a complication related to this dramatic tumor volume reduction in aggressive skull base meningiomas.

The authors describe the case of a 39-year-old woman who had undergone repeat surgical interventions and courses of radiotherapy over the previous 11 years for recurrent cranial and spinal meningiomas. Despite 4 operations over the course of 4 years on her right petroclival meningioma with cavernous sinus and jugular fossa extensions, she had progressive neurological deficits and tumor recurrences. The specimen histology progressed from WHO Grade I initially to Grade II at the time of the third recurrence. The lesion was then irradiated 3 times using stereotactic radiosurgery for further recurrences. More recently, the tumor size increased rapidly on imaging, in association with progressive neurological symptoms arising from brainstem compression and vasogenic edema. Institution of sunitinib therapy yielded a dramatic radiographic response, with marked reduction in the tumor volume and reduction of brainstem vasogenic edema within a few weeks of initiation of treatment. The significant radiographic response of tumor in the clival region was also associated with CSF rhinorrhea from a dural breach created by resolution of the invasive skull base meningioma, which necessitated withholding the sunitinib medication. To address the leak, the authors undertook surgical exploration and transsphenoidal packing using an autologous fat graft and a vascularized pedicled nasoseptal flap. The patient has done well during follow-up of 3 months after packing, with no evidence of recurrent CSF leak, and the medication was subsequently restarted.

Prior clinical data and the dramatic radiographic response in this patient suggest that sunitinib holds promising therapeutic potential in carefully selected patients with recurrent atypical meningiomas where conventional strategies have been exhausted. There is a potential risk of associated CSF rhinorrhea, especially in more invasive skull base lesions showing dramatic radiographic response.

https://thejns.org/doi/abs/10.3171/2016.9.JNS161629

KEY WORDS sunitinib; recurrent atypical meningioma; cerebrospinal fluid rhinorrhea; radiographic response; oncology

$\mathrm{M}$ ENINGIOMAS account for approximately one-third of the total intracranial tumor burden and are the most common type of primary brain tumor. ${ }^{13}$ According to the current epidemiological data, their incidence is 7.7 per 100,000 population, ${ }^{8}$ with a female-tomale ratio of 3.5:1. ${ }^{6}$ Approximately $20 \%$ of meningiomas are atypical (WHO Grade II) with evidence of brain invasion or aggressive histological features, such as increased mitotic activity, the presence of nuclear atypia, and necrosis. ${ }^{9}$ Their aggressive biological behavior is reflected by a roughly 8-fold greater risk of recurrence compared with benign WHO Grade I meningiomas and by the consider-

ABBREVIATIONS OS = overall survival; PDGFR = platelet-derived growth factor receptor; $\mathrm{PFS}$ = progression-free survival; PI-3 K/AKT = phosphatidyl inositol-3 kinase; Raf-MAPK = mitogen-associated protein kinase; SRS = stereotactic radiosurgery; VEGF = vascular endothelial growth factor; VEGFR = VEGF receptor.

SUBMITTED June 20, 2016. ACCEPTED September 7, 2016.

INCLUDE WHEN CITING Published online December 9, 2016; DOI: 10.3171/2016.9.JNS161629. 
ably increased risk of mortality in atypical meningiomas in age- and sex-matched controls. ${ }^{9}$ Recurrence rates of atypical meningioma can exceed 50\%., ${ }^{5,9}$ Currently, the management options for meningioma include surgery and radiation therapy primarily, with only a limited role for medical management (chemotherapy/targeted therapy). ${ }^{3}$ This approach is effective in achieving tumor control of most WHO Grade I meningiomas as well as for a subset of patients with WHO Grade II tumors; 3,13 however, treatment options are limited for patients with higher-grade, inoperable, or recurrent tumors, where conventional therapies of surgery and radiotherapy have been exhausted. Medical management including chemotherapy and targeted therapy are reserved for such cases, ${ }^{3,13}$ although the use of targeted therapy (e.g., sunitinib, vatalanib, bevacizumab) remains largely empirical without robust clinical evidence. A recent single-arm, prospective, Phase II clinical trial is the first to suggest activity of sunitinib in recurrent or aggressive meningiomas.?

Sunitinib malate is an orally administered, small-molecule multiple tyrosine kinase inhibitor that targets vascular endothelial growth factor receptor (VEGFR), platelet-derived growth factor receptor (PDGFR), FMS-like tyrosine kinase, tyrosine protein kinase KIT, macrophage colonystimulating factor 1 receptor, and RET proto-oncogene, most of which are abundantly expressed in meningiomas. ${ }^{5,7}$ It has antiangiogenic, cytostatic, and antimigratory activity for meningioma tumor cells..$^{1,5,7}$ In particular, previously irradiated meningioma cells are more sensitive to adjuvant sunitinib therapy. ${ }^{1,5}$ Because of its impact on both tumor vascularization and proliferation and its superior pharmacological properties as compared with other receptor tyrosine kinase inhibitors, it has become a promising candidate for targeted tumor therapy ${ }^{1,4}$ as highlighted by a recent Phase II clinical trial reported by Kaley et al. ${ }^{5}$ We describe a case in which sunitinib used for treatment of a recurrent atypical meningioma after multiple surgical and radiation treatments was associated with a dramatic reduction in tumor burden.

\section{Case Report}

\section{Clinical Presentation}

This 39-year-old woman had a history of numerous recurrent craniospinal meningiomas since 2005, for which she had received multiple surgical interventions and adjuvant radiotherapy over the past 11 years. She initially presented with diplopia, and subsequently she manifested symptoms of right 5th and 7th cranial nerve dysfunction and lower cranial nerve pareses, with the progression of her recurrent right petroclival meningioma with cavernous sinus and jugular fossa extension. There was no prior history of neurofibromatosis or any familial predisposition of multiple meningiomas. She underwent 4 resections for this tumor in a span of 4 years via translabyrinthine, transcochlear, extradural middle fossa, and extended endonasal transsphenoidal approaches. Subsequently, over the next 4 years, this lesion was irradiated 3 times using stereotactic radiosurgery (SRS) for the progression of the meningioma from WHO Grade I to WHO Grade II by the third recurrence.

\section{Everolimus Therapy: Rationale, Dosing, and Response}

Follow-up imaging 18 months after the last dose of SRS demonstrated stable disease in the right petroclival region (Fig. 1A and B). Repeat brain imaging performed approximately 1 year later demonstrated a marginal increase in recurrent tumor volume without significant worsening of neurological symptoms (Fig. 1C and D). Because the patient had already undergone multiple resections and radiation treatments, an optimal target for possible medical management was sought. To evaluate this, an atypical meningioma specimen from the third recurrence was genetically analyzed. A TSC1 mutation was identified in the tumor, and treatment with $5 \mathrm{mg} /$ day everolimus was started. The patient began to notice a return of intermittent double vision over the course of the next couple of months, which progressed to constant double vision. The patient was given a course of steroids, which initially helped. Over the course of a few more months, she started noticing some right-sided cheek and chin numbness and an increasing right facial droop. She has had right vocal cord paralysis and absence of hearing on the right side since her second surgery.

\section{Sunitinib Therapy: Rationale, Dosing, and Schedule}

Follow-up imaging 6 months after the initiation of everolimus demonstrated a significant increase in tumor size along the right petroclival region with brainstem compression and associated vasogenic edema (Fig. 1E and F). To address the new-onset symptoms and persistent progression of disease on repeat imaging, the everolimus protocol was stopped, and the patient was switched to $50 \mathrm{mg} /$ day of sunitinib in a 4 weeks on, 2 weeks off schedule, as per the protocol described by Kaley et al. ${ }^{5}$

\section{Sunitinib Therapy: Clinical Outcome, Complications, and Radiographic Response}

Clinically, the patient had an improvement in neurological symptoms within a few weeks of initiation of treatment. She soon developed nausea, weakness, and fatigue and had noticeably increased neck and shoulder pain and new knee pain. Additionally, she developed thrombocytopenia. She also developed transient CSF rhinorrhea, which resolved with lumbar drain placement. The sunitinib was withheld after a total duration of 15 days, and the symptoms, presumed to be drug induced, improved within a week.

Radiographically, there appeared to be significant reduction in tumor volume in the right cerebellopontine angle as well as decreased edema in the right cerebellum and pons, most consistent with a positive response to the sunitinib (Fig. $1 \mathrm{G}$ and $\mathrm{H}$ ). She was restarted on an adjusted dose $(37.5 \mathrm{mg} / \mathrm{day})$ of sunitinib, but within a week she again developed CSF rhinorrhea without evidence of meningitis. After a CT cisternogram demonstrated the CSF leak along the clival region (Fig. 2) apparently created by resolution of the invasive skull base meningioma, we undertook surgical exploration via an endoscopic endonasal approach. The clival dural defect was packed transsphenoidally using an autologous abdominal fat graft and vascularized pedicled nasoseptal flap.

The patient had an uneventful recovery, and she was re- 

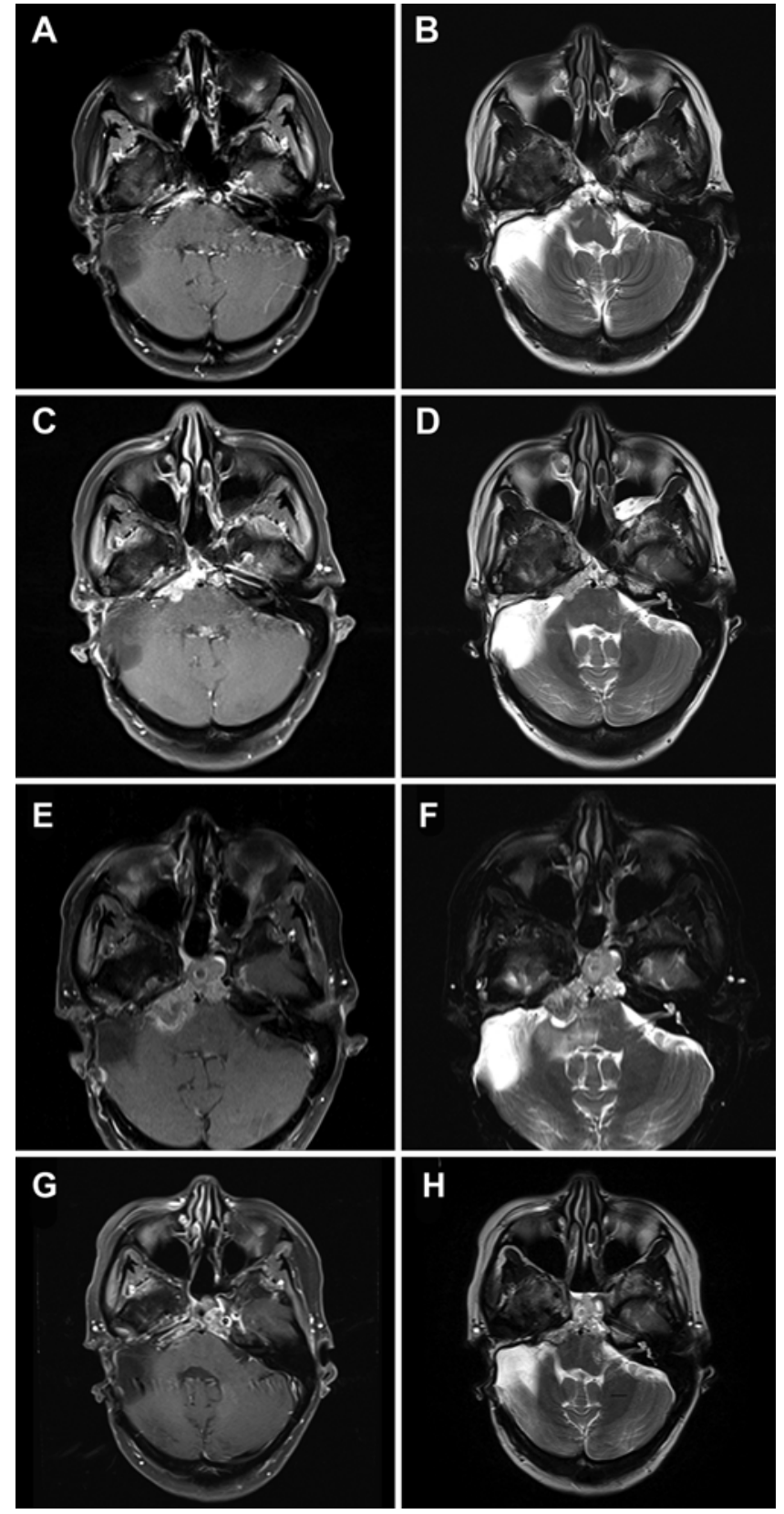

FIG. 1. Axial gadolinium-enhanced (A) and T2-weighted (B) MR images of the brain 18 months after the last dose of SRS, demonstrating subtle linear enhancement measuring $14 \times 6 \mathrm{~mm}$ along the right petroclival dura with no evidence of brainstem compression or vasogenic edema. Follow-up axial gadolinium-enhanced (C) and T2-weighted (D) MR images of the brain obtained approximately 1 year later, demonstrating marginally increased nodular enhancement measuring $29 \times 13 \mathrm{~mm}$ along the right petroclival dura with no evidence of significant brainstem compression or vasogenic edema. Axial gadolinium-enhanced $(E)$ and T2-weighted (F) MR images of the brain obtained after 6 months of treatment with everolimus, demonstrating significantly increased nodular enhancing recurrent tumor measuring $42 \times 23 \mathrm{~mm}$ along the right petroclival dura with evidence of increased brainstem compression and associated vasogenic edema in the brainstem and cerebellar peduncles. Axial gadolinium-enhanced $(\mathbf{G})$ and T2-weighted $(\mathbf{H})$ MR images of the brain after 2 weeks of treatment with sunitinib, demonstrating significantly decreased linear enhancement measuring $13 \times 7 \mathrm{~mm}$ along the right petroclival dura, with no evidence of residual brainstem compression and marked reduction of vasogenic edema in the brainstem and cerebellum.

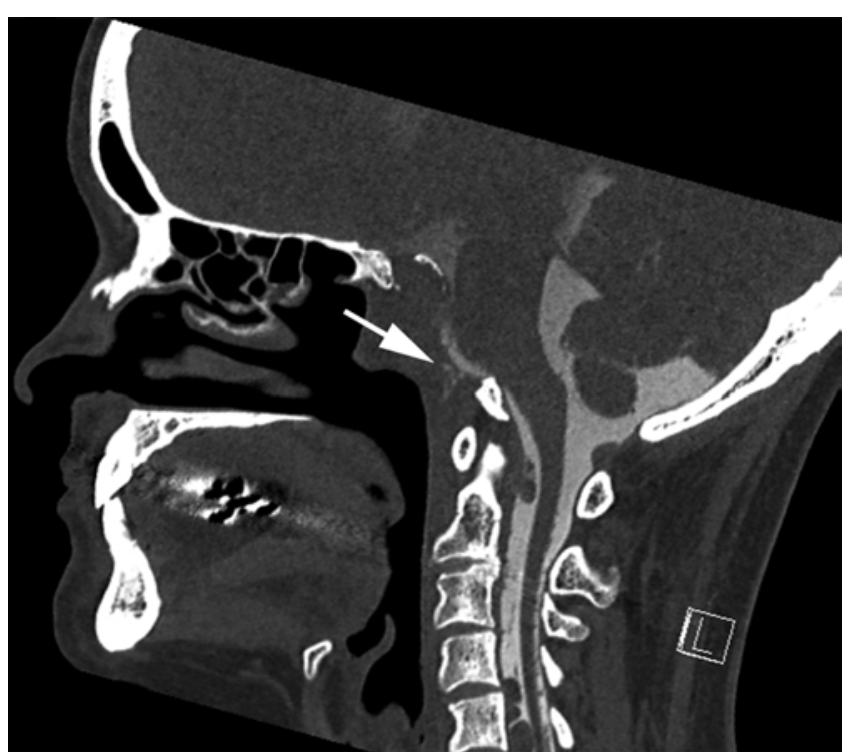

FIG. 2. Sagittal CT cisternogram obtained after resumption of transiently halted sunitinib therapy, showing the largest area of contrast agent leakage (arrow) arising from the prepontine cistern just inferior to the posterior aspect of the sella turcica. The contrast agent pooled at the level of the previously resected sphenoid sinus posterior wall. There are additional foci of contrast enhancement extending anteriorly from the prepontine cistern into the submucosal nasopharyngeal soft tissues located just inferiorly adjacent to the clival remnant.

started on an adjusted dose of sunitinib initially $(37.5 \mathrm{mg} /$ day) that was subsequently increased back to a full dose (50 mg/day). At her last follow-up 3 months after surgical packing, she was doing well and tolerating the medication well. The patient reported that her neck pain has improved approximately $50 \%$. She denies any symptoms of the CSF leak. She continues to have neurological deficits from her prior surgical treatments. She has had significant improvement in her diplopia over the last few months, mostly with near vision improvement. She denies any change to her voice or her ability to swallow, or an increase in cough or drooling. She is now able to close her right eye fully.

\section{Histopathology and Genomic Analysis}

Initial histopathology from the second and third surgeries revealed a meningothelial meningioma (WHO Grade I), with moderate cellularity. The neoplastic cells had round-to-oval, bland nuclei and moderate amounts of eosinophilic cytoplasm. No significant mitotic figures or cytological atypia was identified. The neoplasm infiltrated into the fibrocollagenous tissue. The histology of the right petroclival meningioma had progressed from WHO Grade I to II by the time of the third recurrence (fourth surgery). Histological examination showed pieces of a meningothelial neoplasm of moderate cellularity arranged in sheets. The neoplastic cells exhibited round-to-oval nuclei with a moderate amount of eosinophilic cytoplasm with indistinct cell borders. There were more mitotic figures with approximately 11 per $10 \mathrm{hpf}$ observed (Fig. 3A and inset). Small foci of tumor necrosis were also identified, satisfying the criteria for atypical WHO Grade II meningioma. Immunohistochemistry for VEGF-C was positive throughout the WHO Grade II tumor (Fig. 3B). 

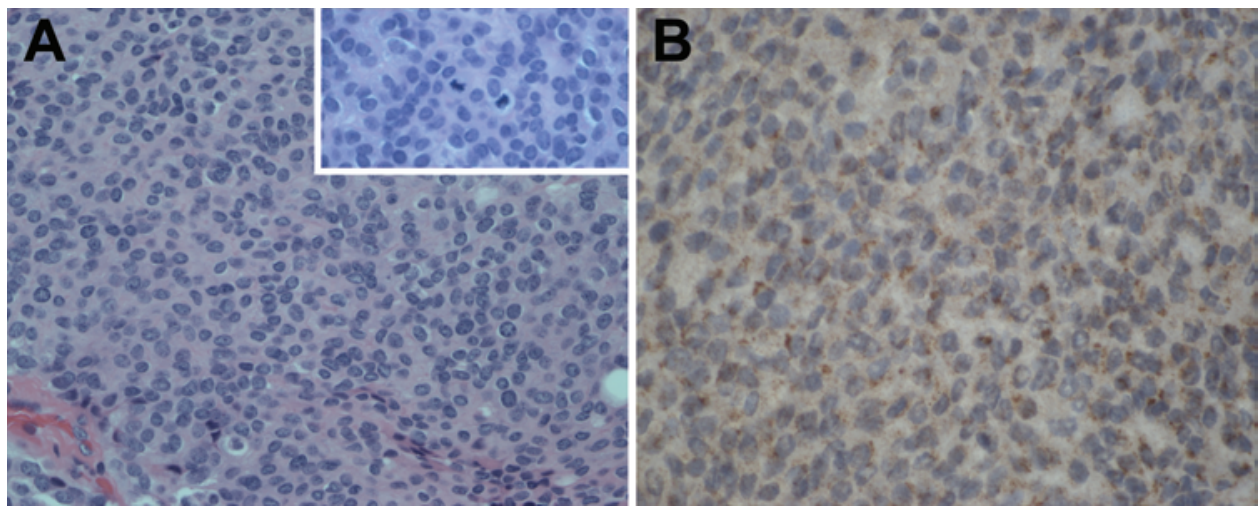

FIG. 3. Histopathological examination of the paraclinoid resection specimen from the fourth surgery, demonstrating meningothelial tumor cells with mild nuclear pleomorphism arranged in sheets (A). Mitoses were easily identified (inset). Immunohistochemistry obtained after genomic analysis demonstrating VEGF-C positivity in the tumor (B). H \& E (A and inset); VEGF immunostain (B). Original magnification $\times 400(A$ and $B$ ) and $\times 600$ (inset). Figure is available in color online only.

When there was evidence of recurrence of tumor despite adequate surgical and radiation treatments, the specimen from the fourth surgery was sent for genomic analysis (Foundation One) to assess for possible genetic mutations for targeted therapy. The findings were suggestive of 2 genomic alterations: TSC1 Y192fs*1 and BAP1 Q684. Everolimus was used as a targeted tumor therapy based on this TSC1 mutation identified on gene sequencing.

\section{Discussion}

We describe a patient in whom sunitinib was used for treatment of a recurrent atypical meningioma after multiple surgical interventions and radiation treatments had failed. A dramatic radiographic response was noted within a few weeks of initiation of treatment, with significant reduction in tumor volume; however, there was CSF rhinorrhea from a dural breach in the clival region, apparently created by resolution of the invasive skull base meningioma. This necessitated transiently withholding sunitinib and completing surgical exploration and transsphenoidal packing using an autologous fat graft and vascularized pedicled nasoseptal flap to seal off the defect.

\section{Pathogenesis in Meningiomas}

In contrast to our improving understanding of the molecular pathogenesis and tumor biology of gliomas, the pathogenetic mechanisms and critical molecular changes involved in meningioma growth and progression are less well understood. ${ }^{3}$ Overexpression of various growth factors (including PDGF, epidermal growth factor, and VEGF), their receptors (PDGFR, epidermal growth factor receptor, and VEGFR), and signal transduction pathways (mitogen-associated protein kinase [Raf/MAPK], phosphatidyl inositol-3 kinase [PI-3 K/AKT], and protein kinase $C$ ) has been implicated, but the relative significance of each is largely unknown. ${ }^{3}$ Histologically, the progression of WHO Grade I meningiomas to Grade II meningiomas with recurrence can be confirmed in 17\%-38\% of patients. ${ }^{7}$ At the cytogenetic level, a stepwise acquisition of chromosomal gains and losses during meningioma progression has been proven. Loss of $1 \mathrm{p}, 6 \mathrm{q}, 10 \mathrm{q}, 14 \mathrm{q}$, and
$18 \mathrm{q}$ and gain of 1q, 9q, 12q, 15q, 17q, and 20q have been proposed as important events in meningioma progression and recurrence. ${ }^{7}$ Radiation-induced aggressive meningiomas demonstrate cytogenetic aberrations in $1 \mathrm{p}, 6 \mathrm{q}$, and chromosome 22 more frequently?

\section{Rationale for Anti-VEGFR/PDGFR Targeted Therapy (sunitinib) in Aggressive Meningiomas}

Evidence from several studies suggests a role for antiangiogenic agents in the treatment paradigm of aggressive and recurrent meningiomas. Upregulation of angiogenic pathways and pathological neovascularization have been demonstrated repeatedly in aggressive meningiomas, especially in the perinecrotic tumor cells potentially experiencing hypoxia, thus providing a pathobiological rationale for such agents. ${ }^{2,12}$ Under hypoxic conditions, VEGF is upregulated via hypoxia-inducible factor in tumor cells and mediates endothelial cell proliferation by binding to its main receptors (VEGFR-1 and -2), leading to an activation of PI-3 K/v-AKT and Raf-MAPK pathways., ${ }^{2,12}$ Additionally, VEGF expression is seen in approximately $83 \%$ of WHO Grade II meningiomas (much higher than benign counterparts) and has been linked to proliferation, migration, vascularity, invasiveness, and recurrence of aggressive meningiomas, which supports the rationale for off-label clinical use of anti-VEGF agents. ${ }^{1-3,10,11}$

In addition to VEGF, PDGF signaling indirectly regulates angiogenesis. ${ }^{10}$ Yang and $\mathrm{Xu}^{14}$ demonstrated that nearly all meningiomas express PDGFR, and its expression is markedly increased in atypical (WHO Grade II) meningiomas. VEGF expression increases with tumor grade (2-fold in atypical and 10-fold in malignant phenotypes compared with benign variants). ${ }^{13}$ VEGF plays a central role in tumor angiogenesis, and there is a growing body of literature indicating that inhibition of VEGF/ VEGFR can lead to significant antitumoral effects. ${ }^{2,3,10}$ Inhibitors of VEGFR, such as sunitinib and sorafenib, have prolonged survival in patients with renal cell carcinoma and gastrointestinal stromal tumors.,13 VEGF also plays a central role in the formation of peritumoral edema, which adds to the morbidity of these tumors. Inhibitors of VEGFR tend to improve clinical symptoms by decreasing peritumoral edema. ${ }^{2,5,10,11}$ 
Radiographic Response and Outcomes With Sunitinib Therapy in Aggressive Meningiomas

In a recent prospective, multicenter, single-arm, Phase II clinical trial, Kaley et al. ${ }^{5}$ evaluated the role of sunitinib in a primary cohort of 36 patients with recurrent WHO Grade II/III meningiomas that were refractory to traditional surgical and radiation therapy options. Sunitinib was administered at a dose of $50 \mathrm{mg} /$ day for Days 1-28 of a 42-day cycle. Progression-free survival (PFS) at 6 months was $42 \%$. The median PFS was 5.2 months, and the median overall survival (OS) was 24.6 months. Expression of VEGFR-2 predicted PFS, with a median PFS of 1.4 months in VEGFR-2-negative patients and 6.4 months in VEGFR-2-positive patients $(\mathrm{p}=0.005)$. Among the 35 patients for whom radiographic response data were available, 1 patient achieved a complete radiographic response, and another had a partial response (both with atypical meningiomas); the majority had stable disease $(\mathrm{n}=25)$, and approximately one-quarter had progressive disease $(n=8)$. Interestingly, the only 2 cases of radiographic reduction of tumor burden with sunitinib therapy in their cohort were noted in patients with atypical tumors. The authors also demonstrated that there was a decrease in perfusion ratios in all patients, suggesting that sunitinib did reach those tumors and exerted an effect on the tumor vasculature.

Our patient with atypical meningioma also had a dramatic reduction in tumor volume with sunitinib therapy within a few weeks of initiation of treatment. The rationale for utilizing sunitinib in this case was essentially empirical, without a clear molecular or genetic basis. Interestingly, everolimus did not have any clear clinical or radiological benefit despite the identified TSC1 mutation observed on tumor gene sequencing, and it had to be discontinued for lack of effect. As a matter of fact, tumor progression was more rapid after everolimus therapy was started. A possible explanation for such an observation is that tumors often evade the blocked receptors after targeted therapy, using alternative mechanisms of survival and progression. Therefore, single targeted therapeutic agents may not achieve the expected goals in refractory and recurrent tumors, but adding an additional targeted agent with a different mechanism of action may allow them to synergize and reduce the tumor growth drastically, as was observed in our patient. Therefore, multitargeted therapy tailored to molecular and genetic bases, if applicable, holds a promising future toward managing such refractory and recurrent tumors.

Yoshikawa et al. ${ }^{15}$ described another case in which a proteomic analysis in a patient with a malignant meningioma identified highly expressed PDGFR- $\beta$. On the basis of this observation, $25 \mathrm{mg} /$ day of sunitinib was administered using the same protocol of 4-week therapy followed by a 2 -week rest period. The authors noted that PDGFR- $\beta$ plays a major role in meningioma cell proliferation through an autocrine loop system, especially in higher-grade meningiomas. In this case, microvascular density, a surrogate marker of angiogenesis, was reduced by approximately one-third after sunitinib was administered. The tumor showed necrosis after treatment was initiated but progressed rapidly after treatment was stopped, and the patient died.

\section{Complications of Sunitinib Therapy in Patients With Aggressive Meningiomas}

In the clinical trial by Kaley et al., ${ }^{5}$ the authors observed an overall toxicity of one Grade 5 intratumoral hemorrhage, two Grade 3 and one Grade 4 CNS/intratumoral hemorrhages, one Grade 3 and one Grade 4 thrombotic microangiopathy, and one Grade 3 gastrointestinal perforation with sunitinib therapy. This study also had a secondary (exploratory) cohort of 13 patients with a diagnosis of WHO Grade I meningioma, higher-grade hemangiopericytoma, or WHO Grade I hemangioblastoma in which no significant activity was noticed with sunitinib therapy. Of the 49 patients enrolled in that study, a total of 16 patients $(32 \%)$ required dose reduction, and 11 patients $(22 \%)$ had to discontinue sunitinib because of toxicity and were removed from the study. Despite the apparent efficacy, considerable toxicity with sunitinib was noted. Overall, the rate of CNS hemorrhage was low (8\%) and comparable with rates reported in other angiogenesis inhibitor studies in patients with gliomas. Similarly, the sunitinib therapy had to be discontinued because of drug-induced pancytopenia in the patient reported by Yoshikawa et al. ${ }^{15}$

Our patient also had transient thrombocytopenia requiring a dose reduction initially. This is the first reported case of CSF rhinorrhea as a complication of sunitinib therapy. Once the dural breach was packed, the patient resumed the full dose of sunitinib and is tolerating it reasonably well. Drug intolerance is a major limitation while using targeted therapies, and the dosage needs to be individually tailored according to the tolerance and benefit observed.

\section{Conclusions}

This case report highlights the potential role of sunitinib for patients with recurrent atypical meningioma and also highlights that CSF rhinorrhea is a potential complication of this therapy, especially in more invasive skull base lesions showing a dramatic radiographic response. Sunitinib appears to hold promising therapeutic potential in carefully selected patients with recurrent atypical meningiomas where conventional strategies have been exhausted. Whether these effects will be translated into improved natural history of the disease progression with improved PFS and OS is yet to be determined.

\section{Acknowledgments}

We thank Kristin Kraus, MSc, our medical editor, for her contribution to manuscript editing.

\section{References}

1. Andrae N, Kirches E, Hartig R, Haase D, Keilhoff G, Kalinski T, et al: Sunitinib targets PDGF-receptor and Flt3 and reduces survival and migration of human meningioma cells. Eur J Cancer 48:1831-1841, 2012

2. Baumgarten P, Brokinkel B, Zinke J, Zachskorn C, Ebel H, Albert FK, et al: Expression of vascular endothelial growth factor (VEGF) and its receptors VEGFR1 and VEGFR2 in primary and recurrent WHO Grade III meningiomas. Histol Histopathol 28:1157-1166, 2013

3. Chamberlain MC, Barnholtz-Sloan JS: Medical treatment of 
recurrent meningiomas. Expert Rev Neurother 11:14251432, 2011

4. Chow LQ, Eckhardt SG: Sunitinib: from rational design to clinical efficacy. J Clin Oncol 25:884-896, 2007

5. Kaley TJ, Wen P, Schiff D, Ligon K, Haidar S, Karimi S, et al: Phase II trial of sunitinib for recurrent and progressive atypical and anaplastic meningioma. Neuro Oncol 17:116121,2015

6. Klaeboe L, Lonn S, Scheie D, Auvinen A, Christensen HC, Feychting M, et al: Incidence of intracranial meningiomas in Denmark, Finland, Norway and Sweden, 1968-1997. Int J Cancer 117:996-1001, 2005

7. Mawrin C, Chung C, Preusser M: Biology and clinical management challenges in meningioma. Am Soc Clin Oncol Educ Book 2015:e106-e115, 2015

8. Ostrom QT, Gittleman H, Farah P, Ondracek A, Chen Y, Wolinsky Y, et al: CBTRUS statistical report: Primary brain and central nervous system tumors diagnosed in the United States in 2006-2010. Neuro Oncol 15 (Suppl 2):ii1-ii56, 2013

9. Pearson BE, Markert JM, Fisher WS, Guthrie BL, Fiveash JB, Palmer CA, et al: Hitting a moving target: evolution of a treatment paradigm for atypical meningiomas amid changing diagnostic criteria. Neurosurg Focus 24(5):E3, 2008

10. Pfister C, Pfrommer H, Tatagiba MS, Roser F: Vascular endothelial growth factor signals through platelet-derived growth factor receptor $\beta$ in meningiomas in vitro. Br J Cancer 107:1702-1713, 2012

11. Pietsch T, Valter MM, Wolf HK, von Deimling A, Huang HJ, Cavenee WK, et al: Expression and distribution of vascular endothelial growth factor protein in human brain tumors. Acta Neuropathol 93:109-117, 1997

12. Shweiki D, Itin A, Soffer D, Keshet E: Vascular endothelial growth factor induced by hypoxia may mediate hypoxiainitiated angiogenesis. Nature 359:843-845, 1992
13. Wen PY, Quant E, Drappatz J, Beroukhim R, Norden AD: Medical therapies for meningiomas. J Neurooncol 99:365378, 2010

14. Yang SY, Xu GM: Expression of PDGF and its receptor as well as their relationship to proliferating activity and apoptosis of meningiomas in human meningiomas. J Clin Neurosci 8 (Suppl 1):49-53, 2001

15. Yoshikawa A, Nakada M, Ohtsuki S, Hayashi Y, Obuchi W, Sato Y, et al: Recurrent anaplastic meningioma treated by sunitinib based on results from quantitative proteomics. Neuropathol Appl Neurobiol 38:105-110, 2012

\section{Disclosures}

Dr. Colman reports the following: consultant for Hoffman La Roche, Upsher-Smith, Genentech, Novocure, Oxigene, CytRx, Omniox, and Insys.

\section{Author Contributions}

Conception and design: Couldwell, Raheja. Acquisition of data: Raheja, Palmer. Analysis and interpretation of data: Palmer. Drafting the article: Raheja. Critically revising the article: Couldwell, Colman, Palmer. Reviewed submitted version of manuscript: all authors. Approved the final version of the manuscript on behalf of all authors: Couldwell.

\section{Correspondence}

William T. Couldwell, Department of Neurosurgery, Clinical Neurosciences Center, University of Utah, 175 N Medical Dr. East, Salt Lake City, UT 84132. email: neuropub@hsc.utah.edu. 